EPJ Web of Conferences 81, 01025 (2014)

DOI: $10.1051 /$ epjconf/ 20148101025

(C) Owned by the authors, published by EDP Sciences, 2014

\title{
Eta Meson Production in Proton-Deuteron Collisions
}

\author{
Alfons Khoukaz ${ }^{1, a}$ \\ ${ }^{1}$ Institut für Kernphysik, Westfälische-Wilhelms-Universität Münster, 48149 Münster, Germany
}

\begin{abstract}
Since many years the strong final state interaction between $\eta$-mesons and light nuclei and the discussed possibility for the formation of $\eta$-mesic nuclei has attracted broad experimental and theoretical investigations. Prominent examples are production experiments on the ${ }^{3} \mathrm{He}-\eta$ final state which present an unexpected strong total cross section enhancement at threshold, indicating the presence of a pole very close to threshold. Stimulated by these results new proton-deuteron collision experiments on the $\eta$ meson production have been performed in the near-threshold region and at higher excess energies using both polarized and unpolarized beams. Recent results are presented and discussed in this contribution.
\end{abstract}

\section{Introduction}

Studies on the $p d \rightarrow{ }^{3} \mathrm{He} \eta$ or $d p \rightarrow{ }^{3} \mathrm{He} \eta$ reaction are motivated by the very anomalous results near threshold [1-4] as well as by the poorly understood production mechanism. It was suggested that the rapid jump of the total cross section as function of the excess energy $Q$ is an effect of a surprisingly strong final state interaction (FSI) in the $\eta^{3} \mathrm{He}$ system that might lead to a quasi-bound $\eta$-nuclear state [5]. Detailed studies on former measurements with unpolarized beams and targets show a pole in the $\eta^{3} \mathrm{He}$ elastic amplitude for $|Q|<0.5 \mathrm{MeV}$ [3]. This finding is supported by the analysis of the variation of the angular dependence of the cross section with the excess energy $Q$ [6]. In order to further investigate this system new data with polarized deuteron beams have been taken close to the production threshold at the ANKE spectrometer [7] located at the COSY accelerator of the Forschungszentrum Jülich. These allow for complementary investigations on the ansatz of a strong FSI in the $\eta^{3} \mathrm{He}$ system [8]. Furthermore, data at higher excess energies have been taken using the WASA-at-COSY installation which allow for more detailed investigations on the total and differential cross sections which are of high interest for the study of the dominant production processes [9].

\section{Deuteron tensor analyzing power $t_{20}$ of the $\overrightarrow{d p} \rightarrow{ }^{3} \mathrm{He} \eta$ reaction}

Data very close to the $d p \rightarrow{ }^{3} \mathrm{He} \eta$ production threshold support the assumption of the $\eta^{3} \mathrm{He}$ system being in a relative $s$-wave for $|Q|<4 \mathrm{MeV}$ [3]. According to the quantum numbers of the participating particles this $s$-wave can be caused either by the total spin $S=\frac{3}{2}$ or $S=\frac{1}{2}$ of the initial states and a possible different energy behavior of these states will influence measurements of the deuteron tensor analyzing power $t_{20}$ of the $\vec{d} p \rightarrow{ }^{3} \mathrm{He} \eta$ reaction. The assumption of a pure $s$-wave FSI would require

\footnotetext{
a e-mail: khoukaz@uni-muenster.de
} 
that the tensor analyzing power $t_{20}$ as function of the excess energy should be constant although the unpolarized total cross section show the reported strong energy variation. In order to investigate this in more detail the deuteron tensor analyzing power $t_{20}$ of the $\vec{d} p \rightarrow{ }^{3} \mathrm{He} \eta$ reaction has been studied from threshold up to an excess energy of $Q=11 \mathrm{MeV}$ using the ANKE experiment [8]. The new data are consistent with a constant value of $t_{20}=-0.21 \pm 0.02 \pm 0.05$ and are presented in figure 1 together with former results from Saclay [1].
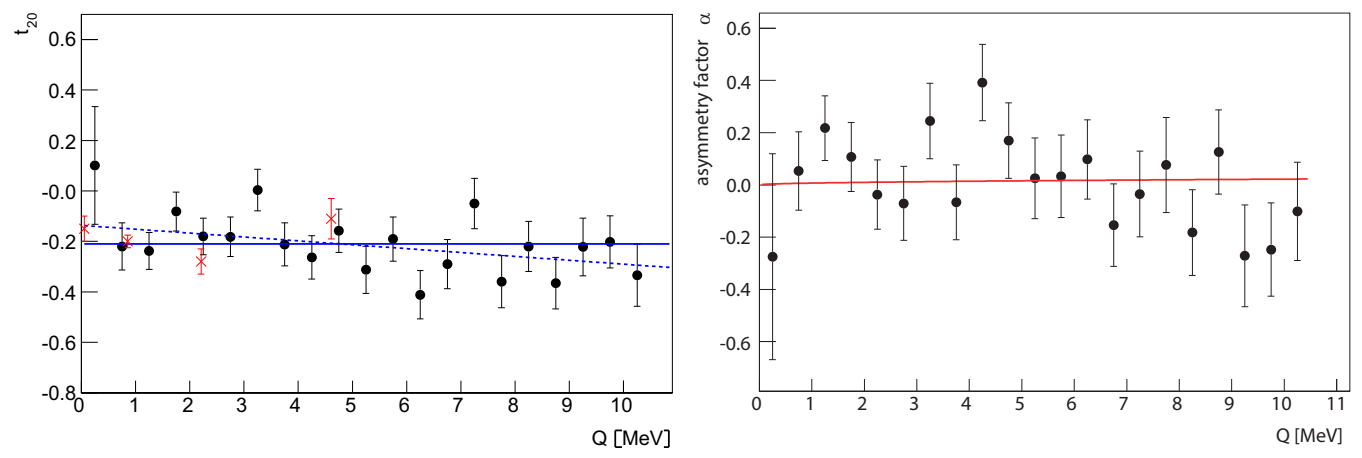

Figure 1. Recent results from the ANKE collaboration. Left: Tensor analysing power $t_{20}$ (black circles) measured for the $\vec{d} p \rightarrow{ }^{3} \mathrm{He} \eta$ reaction. The solid and dashed lines are, respectively, constant and linear fits to these data. Also shown are former results from Saclay [1] (red crosses). Right: Energy dependence of the asymmetry factor $\alpha$ of Eq. (2), fitted as a function of the ${ }^{3} \mathrm{He}$ c.m. momentum. The figures were adapted from [8].

However, in order to study additional energy dependent effects in the $\eta^{3} \mathrm{He}$ system different from FSI, which might depend on the spin configuration in the initial state, one might allow for a linear dependence of $t_{20}$ on $Q$, resulting in [8]

$$
t_{20}=(-0.14 \pm 0.04)+(-0.02 \pm 0.01) Q .
$$

Former precision data obtained with unpolarized targets and beams [3, 4] showed for $Q>4 \mathrm{MeV}$ a significant linear dependence of the $d p \rightarrow{ }^{3} \mathrm{He} \eta$ differential cross section on $\cos \theta$, with $\theta$ as the c.m. polar angle of the ${ }^{3} \mathrm{He}$ with respect to the deuteron beam direction. The new data from ANKE allow for additional information, i.e. the angular dependence of $t_{20}$, which can be expressed by the asymmetry parameter through

$$
\alpha=\mathrm{d} t_{20} /\left.\mathrm{d} \cos \theta\right|_{\cos \theta=0} .
$$

The resulting values of $\alpha$ are presented in figure 1 . The asymmetry parameter itself reflects interferences between odd and even $\eta$ partial waves and is by this an odd function of the $\eta$ c.m. momentum $p_{\eta}$ which, therefore, vanishes at threshold. The data shown in figure 1 are compatible with zero, however, allowing for a linear fit in $p_{\eta}$ results in $\alpha=(0.0002 \pm 0.0005) p_{\eta}$, where $p_{\eta}$ is measured in $\mathrm{MeV} / c$. Obviously there is no sign of any $s-p$ interference, while the unpolarised cross section data show a significant non-isotropy [6].

The gained results can be interpreted more quantitatively by more detailed studies on the production amplitudes of the $d p \rightarrow{ }^{3} \mathrm{He} \eta$ reaction. As presented in Ref. [10] at low excitation energies, or at arbitrary energies in the forward and backward directions, the spin dependence of the production amplitude can be expressed by two independent scalar amplitudes $A$ and $B$ :

$$
f=\bar{u}_{3 \mathrm{He}} \hat{\vec{p}}_{p} \cdot\left(A \vec{\varepsilon}_{d}+i B \vec{\varepsilon}_{d} \times \vec{\sigma}\right) u_{p},
$$


with $u_{3} \mathrm{He}$ and $u_{p}$ as the Pauli spinors, $\vec{\varepsilon}_{d}$ as the polarisation vector of the deuteron, and $\hat{\vec{p}}_{p}$ as direction of the incident proton beam in the c.m. frame. Based on this equation the differential cross section and the deuteron tensor analyzing power may then be written as:

$$
\frac{\mathrm{d} \sigma}{\mathrm{d} \Omega}=\frac{p_{\eta}}{3 p_{p}}\left(|A|^{2}+2|B|^{2}\right) \text { and } t_{20}=\sqrt{2} \frac{|B|^{2}-|A|^{2}}{2|B|^{2}+|A|^{2}} .
$$

From these equation it becomes obvious that an absence of an energy dependence of the angular average of $t_{20}$ directly corresponds to an energy-independent ratio of $|A|^{2} /|B|^{2}$. However, allowing for a possible energy dependence the linear fit in Eq. (1) leads to

$$
|B|^{2} /|A|^{2}=(0.75 \pm 0.06)-(0.014 \pm 0.014) Q .
$$

This result is presented in figure 2 showing the angular averages of the amplitudes $|A|^{2}$ and $|B|^{2}$ extracted from the new ANKE $t_{20}$ data. The striking energy variation of the amplitudes is caused by the strong FSI in the $\eta^{3} \mathrm{He}$ system while a possible energy variation from Eq. (1) is only of minor importance. Moreover, this possible energy variation itself is reported to be only relevant on an excess energy scale of $\approx 50 \mathrm{MeV}$ [8]. Therefore, the rapid cross section increase at threshold within $1 \mathrm{MeV}$ of excess energy can not be explained by significant contributions of such spin dependent effects.

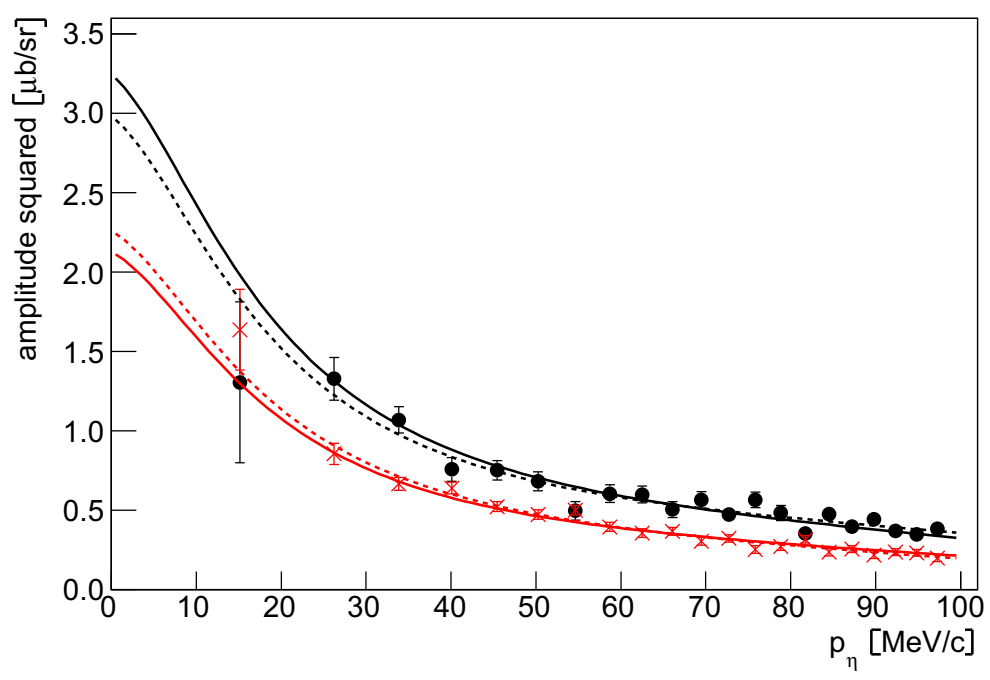

Figure 2. Angular averages of $|A|^{2}$ (black circles) and $|B|^{2}$ (red crosses) extracted from the recently reported ANKE $t_{20}$ results [8]. The solid and the dashed lines are based on results from former unpolarized ANKE measurements [3] and consider a constant value of $t_{20}=-0.21$ (solid line) as well as a possible energy variation (dashed line) of $t_{20}$ (see Eq. (1)). The figure was taken from [8].

Furthermore, the new data from ANKE allow for more detailed information on the structure of the production amplitudes itself. A combined analysis of both the former unpolarized and the new polarized data suggest an ansatz as described in [8]:

$$
\begin{aligned}
A & =A_{0}\left[\operatorname{FSI}\left(p_{\eta}\right)+\alpha p_{\eta} \cos \theta+\beta p_{\eta}^{2}\left(3 \cos ^{2} \theta-1\right) / 2\right] \\
B & =B_{0}\left[\operatorname{FSI}\left(p_{\eta}\right)+\alpha p_{\eta} \cos \theta+\beta p_{\eta}^{2}\left(3 \cos ^{2} \theta-1\right) / 2\right],
\end{aligned}
$$


where the FSI factor only influences the $s$-wave term. Assuming a cancellation of the interference between the $s$ and $d$ waves and the square of the $p$ waves, both the vanishing energy and angular dependence of $t_{20}$ reported in Ref. [8] and the linearity of the (unpolarized) differential cross sections $[3,4]$ could be explained.

\section{Studies on the $p d \rightarrow{ }^{3} \mathrm{He} \eta$ reaction at higher excess energies}

Different to the very near-threshold region of the $p d(d p) \rightarrow{ }^{3} \mathrm{He} \eta$ reaction, for which now a solid data base exists $[1-4,11]$, there are only few data sets at higher energies. Due to this poorly known excitation function only limited information can be extracted from data, e.g. on the underlying production processes. Model calculations available in the literature [12], based on either a one-step or two-step model, fail to explain both the total and differential section of this reaction channel. Due to this, new calculations based on a boson exchange model are proposed for which new precision data at higher excess energies are required [12]. Therefore, recently new data on the $p d \rightarrow{ }^{3} \mathrm{He} \eta$ reaction from the WASA-at-COSY installation at the COSY storage ring have been analyzed in order to provide total and differential cross sections in this regime of excess energies with low uncertainties. For this purpose protons with beam energies of $980 \mathrm{MeV}(Q=48.8 \mathrm{MeV})$ and $1000 \mathrm{MeV}(Q=59.8 \mathrm{MeV})$, respectively, were scattered on a deuterium pellet target [13]. In figure 3 the obtained angular distributions of the emitted $\eta$ meson in the center-of-mass system are presented in arbitrary units including statistical uncertainties and systematic uncertainties except contributions from the overall normalization.

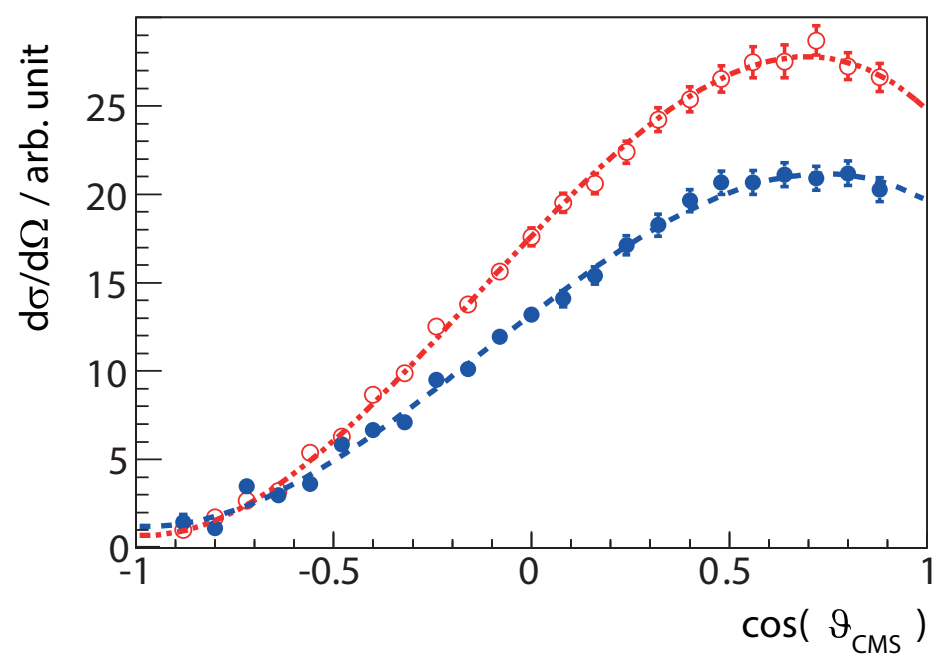

Figure 3. Angular distributions obtained at WASA-at-COSY for the $p d \rightarrow{ }^{3} \mathrm{He} \eta$ reaction at $48.8 \mathrm{MeV}$ (filled circles) and $59.8 \mathrm{MeV}$ data (open circles) in arbitrary units. The figure was adapted from [9].

Obviously both data sets expose a distinct forward-peaked angular asymmetry with almost vanishing backward cross sections, which can be described well by a third-order polynomial fit (dashed lines). Furthermore, these new data allow for the determination of the cross section ratio which was 
MESON 2014 $-13^{\text {th }}$ International Workshop on Production, Properties and Interaction of Mesons

found to be:

$$
\frac{\sigma_{\eta}(48.8 \mathrm{MeV})}{\sigma_{\eta}(59.8 \mathrm{MeV})}=0.77 \pm 0.06
$$

The impact of this ratio can be studied in detail if the new $59.8 \mathrm{MeV}$ data are scaled to the cross section $\sigma(59.4 \mathrm{MeV})=(388.1 \pm 7.1 \pm 58.0) \mathrm{nb}$ obtained at ANKE [14]. With this scaling the new WASA-at-COSY data point with lower excess energy would result in [9]

$$
\sigma(48.8 \pm 0.8 \mathrm{MeV})=(298 \pm 24 \pm 49) \mathrm{nb} .
$$

This situation is illustrated in figure 4, presenting the available cross sections up to an excess energy of $Q=120 \mathrm{MeV}$ in combination with the new WASA-at-COSY results. Note that the discussed scaling requires that both new data at $Q \approx 60 \mathrm{MeV}$ coincide.

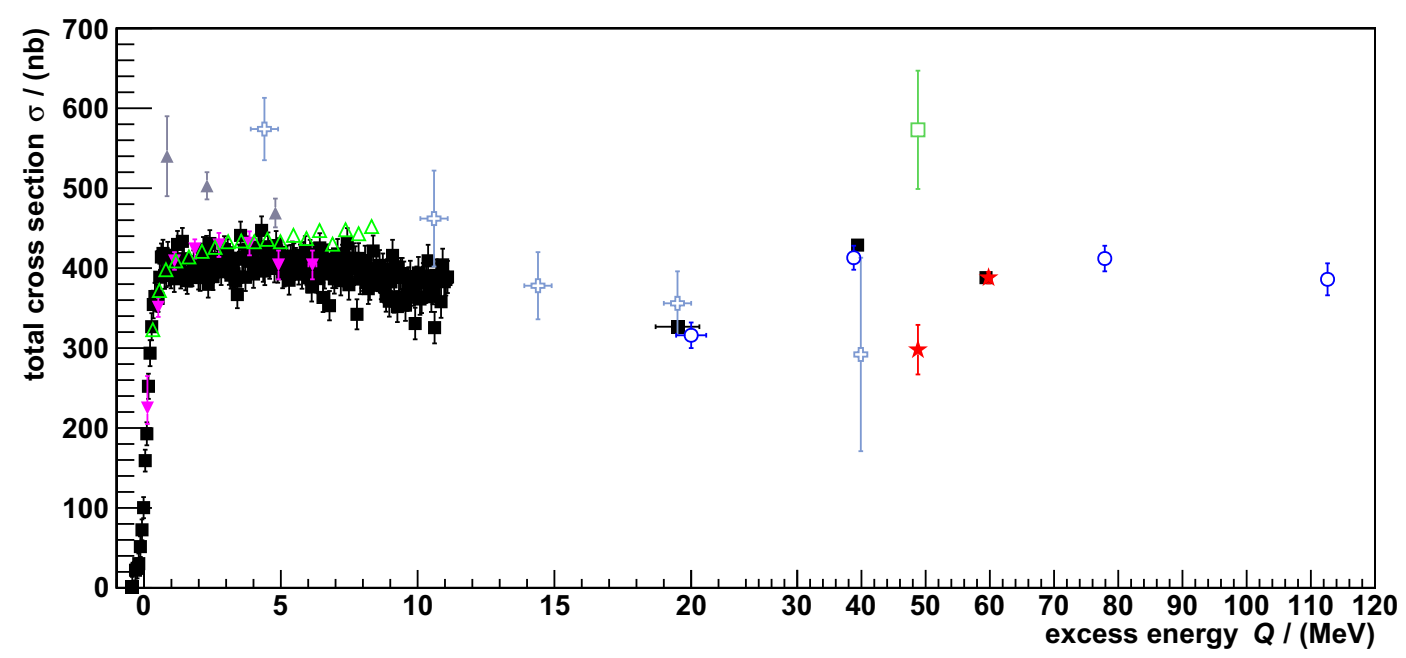

Figure 4. Total cross sections of the reaction $p d \rightarrow{ }^{3} \mathrm{He} \eta$ as function of the excess energy. All data are shown without systematic uncertainties from absolute normalization. The new data from WASA-at-COSY are indicated by red stars and are arbitrarily scaled to the ANKE data point at $\mathrm{Q}=59.4 \mathrm{MeV}$ [14]. In addition, data are shown from ref. [1] (filled gray triangles), ref. [2] (inverted purple triangles), ref. [15] (open green square), refs. [16, 17] (open blue circles), ref. [11] (open light blue crosses), ref. [4] (open green triangles) and refs. [3, 14] (filled black squares). The figure was taken from [9].

From figure 4 it becomes obvious that the new precisely determined cross section ratio from WASA-at-COSY in combination with the existing data from ANKE [14] and WASA/PROMICE [16, 17] indicates the presence of a distinct cross section variation between $Q=20 \mathrm{MeV}$ and $Q=60 \mathrm{MeV}$, which is not smooth. This observation is highly surprising since this effect is unlikely to be caused by a FSI and might indicate either the onset of higher partial waves or a variation of the production mechanism [9]. Therefore, new data with high statistics have been taken very recently in the excess energy region of $Q=20-80 \mathrm{MeV}$ with the WASA-at-COSY installation. In total data at 15 different energies have been recorded which will allow for a detailed investigation of total and differential cross sections with high precision [18]. 


\section{Summary}

New data on the reaction $p d(d p) \rightarrow{ }^{3} \mathrm{He} \eta$ have been reported recently by both the ANKE and WASAat-COSY experiments, both located at the COSY accelerator of the Forschungszentrum Jülich. The data obtained close to the production threshold using polarized deuteron beams enable for new important insights on the relevant production amplitudes and support the earlier findings of a very strong final state interaction in the $\eta^{3} \mathrm{He}$ system. In addition, the new data at higher excess energies show an unexpected cross section variation within a short range of excess energies which might indicate variations of the production processes. Therefore, these new data in combination with very recently recorded data from WASA-at-COSY $[18,19]$ allow for a deeper understanding of this highly interesting meson production reaction.

\section{Acknowledgements}

The support from the FFE Funding Program of the Jülich Center for Hadron Physics is kindly acknowledged.

\section{References}

[1] J. Berger et al., Phys. Rev. Lett. 61, 919 (1988)

[2] B. Mayer et al., Phys. Rev. C 53, 2068 (1996)

[3] T. Mersmann et al., Phys. Rev. Lett. 98, 242301 (2007)

[4] J. Smyrski et al., Phys. Lett. B 649, 258 (2007)

[5] C. Wilkin, Phys. Rev. C 47 (1993) R938

[6] C. Wilkin et al., Phys. Lett. B 654, 92 (2007)

[7] S. Barsov et al., Nucl. Instrum. Methods Phys. Res. Sect. A, Accel. Spectrom. Detect. Assoc. Equip. 462, 364 (1997)

[8] M. Papenbrock et al., Phys. Lett. B 734, 333 (2014)

[9] P. Adlarson et al., Eur. Phys. J. A 50, 100 (2014)

[10] J.-F. Germond, C. Wilkin, J. Phys. G 14 (1988) 181

[11] H.-H. Adam et al., Phys. Rev. C 75, 014004 (2007)

[12] N. G. Kelkar et al., Rep. Prog. Phys. 76, 066301 (2013)

[13] F. Bergmann et al., IKP Annual Report 2008, FZ-Jülich (2008), Jül-4282, p. 52

[14] T. Rausmann et al., Phys. Rev. C 80, 017001 (2009)

[15] M. Betigeri et al., Phys. Lett. B 472, 267 (2000)

[16] R. Bilger et al., Phys. Rev. C 65, 044608 (2002)

[17] R. Bilger et al., Phys. Rev. C 69, 014003 (2004)

[18] A. Khoukaz, 42nd Meeting of the COSY Programme Advisory Committee (PAC), Proposal 220 (2014)

[19] N. Hüsken, doctoral thesis, Westfälische Wilhelms-Universität Münster, Münster, Germany, in preparation 